\title{
COMPRESSION BASED FACE RECOGNITION USING DWT AND SVM
}

\author{
Sujatha $\mathrm{BM}^{1}$ Chetan Tippanna Madiwalar ${ }^{2}$ Suresh Babu $\mathrm{K}^{2}$ Raja K B ${ }^{2}$ and \\ Venugopal K R ${ }^{2}$ \\ ${ }^{1}$ Department of Electronics and Communication Engineering, \\ Acharya Institute of Technology, Bangalore, India \\ ${ }^{2}$ University Visvesvaraya College of Engineering, Bangalore, India
}

\begin{abstract}
The biometric is used to identify a person effectively and employ in almost all applications of day to day activities. In this paper, we propose compression based face recognition using Discrete Wavelet Transform (DWT) and Support Vector Machine (SVM). The novel concept of converting many images of single person into one image using averaging technique is introduced to reduce execution time and memory. The DWT is applied on averaged face image to obtain approximation $(L L)$ and detailed bands. The LL band coefficients are given as input to SVM to obtain Support vectors (SV's). The LL coefficients of DWT and SV's are fused based on arithmetic addition to extract final features. The Euclidean Distance (ED) is used to compare test image features with database image features to compute performance parameters. It is observed that, the proposed algorithm is better in terms of performance compared to existing algorithms.
\end{abstract}

\section{KEYWORDS}

Biometrics, Face Recognition, Discrete Wavelet Transform, Support Vector Machine, Fusion.

\section{INTRODUCTION}

The personnel identification and personal data must be protected from hackers as the technology is advancing day by day. The traditional methods of identifying using ID badges, Passwords and PIN'S etc., are not reliable, since these devices can be lost or stolen. An alternative method to identify a person is Biometrics, which is more reliable as this technique is related to human body parts and behaviour of a person. The biometrics is broadly classified into two groups' viz., physiological biometrics and behavioural biometrics. The physiological biometric traits such as Face, Iris, Palmprint, Fingerprint, DNA etc., have constant characteristics. The behavioural biometric traits such as Signature, Gait, Voice, Keystroke etc., have variable characteristics based on the behaviour of a person. The biometrics provides high level of security by denying access to unauthorized persons. In recent years biometrics is being used in every field of technology such as home security, industries, educational institutes, access to electronic devices to defence areas, preparation of country database, cloud computing, Big data analytics etc.

Face recognition is one of the better physiological biometrics trait to recognize a person for several activities. The face recognition has an advantage compared to other biometric trait recognition, since it does not require any physical interaction or cooperation of a person while acquiring face images. The face recognition system has three sections viz., enrolment section, test 
Signal \& Image Processing : An International Journal (SIPIJ) Vol.7, No.3, June 2016

section and matching section. Face database images are acquired using sensors and features are extracted using spatial or transform domain techniques.

Contribution: In this paper Compression based Face Recognition using DWT and SVM is proposed. The novel concept of converting many images of person into one image is introduced. The face images are compressed using DWT and SVM. The features are extracted by fusing LL band coefficients with Support Vectors of LL band.

Organisation: The remaining sections of this paper are structured as follows. Section 2 explains the literature survey of existing techniques. Section 3 describes the proposed face recognition model. The proposed algorithm is given in section 4. The performance analysis is discussed in section 5.Conclusion is given Section 6.

\section{LITERATURE SURVEY}

In this section, the existing various techniques of biometrics are discussed. Thai Hoang Le et and Len Bui [1] proposed a model on Face Recognition Based on Support Vector Machine (SVM) and 2-Dimensional Principle Component Analysis (2DPCA). In this model feature vectors are obtained using 2DPCA technique and then different classifiers like Multi Layer Perception (MLP), K-Nearest Neighbor (K-NN) and SVM are used for recognition. The experiment result shows that the combination of 2DPCA and SVM yields a greater accuracy. Saeid Fazli et al., [2] proposed JPEG2000 Image Compression using SVM and DWT. Initially the images are DC level shifted by subtracting 128 from each pixel. The shifted images undergo five levels of DWT using 9/7 filter bank to get LL band. Then SVM is applied for further compression ie., SVM selects the desired features out of obtained features and it is called Support Vectors. Finally the images are scalar quantized followed by Huffman encoding. Harin Sellahewa and Sabah A. Jassim [3] proposed Image-Quality-Based Adaptive Face Recognition. This model presents an approach to overcome one of the main constraints like varying lighting conditions. Image quality (Q) is measured in terms of illumination distortion in comparison to known reference image. Reference image is obtained by averaging images of 38 individual faces. Later Global luminance distortion in Q (GLQ) is calculated for each image. If GLQ is less than a predefined threshold, normalization is performed. Later wavelet transform like Pyramid Scheme is applied for feature extraction. At a resolution level of $k$, the pyramid scheme decomposes an image $I$ into $3 k+1$. The highest identification accuracy is achieved by fusing the similarity scores of LH and HL subbands.

Vinoda Yaragatti and Bhaskar [4] proposed face recognition using neural network. In this approach, features of images are obtained by fusing the Dual Tree Complex Wavelet Transform (DTCWT) coefficients and Principle Component Analysis (PCA) coefficients. Finally these features are compared with the features of database which are already trained in Artificial Neural Network (ANN). Idan Ram et al., [5] Proposed Facial Image Compression using Patch-Ordering Based Adaptive Wavelet Transform. A Compression algorithm using Redundant Tree Based Wavelet Transform (RTBWT) is implemented. Images are compressed by applying sparse coding using RTBWT, then quantizing the result and applying the encoding and a post processing filter for further improvement of the results. Sonja Grgic and Grgic [6] Proposed Performance Analysis of Image Compression Using Wavelets. Discrete Wavelet transform based compression technique with different type of the wavelets are used analyze the signal to noise ratio. Also discrete cosine transform compression also considered for the SNR ratio and observing the result. Michael Elad et al., [7] Proposed a Low Bit-Rate Compression of Facial Images. Geometrically deform the image into canonical form by mapping each facial feature into corresponding spatial location. Then images are converted into tiles and model these tiles in a compact manner. Using bit allocation and vector tree quantization a lossy compression is achieved on these tiles, resulting 
Signal \& Image Processing : An International Journal (SIPIJ) Vol.7, No.3, June 2016

very low bit rate output image. Kai Dong-Hyuk Shin et al., [8] proposed using Adaptive Gaussian Filtering. The standard deviation that used for the filter is found out from the input noisy image. For calculating the noise level, image is divided into blocks and selects the smooth blocks. Standard deviation is calculated from difference of smooth blocks of input image and filtered image. Munawar Hayat et al., [9] proposed the deep reconstruction model for image set classification. These are mainly used in networks such as multi-view cameras, personal albums. This paper represents the deep reconstruction of samples given and detects the geometric structures automatically; here they have used Template Deep Reconstruction Model (TDRM), which takes place initialization of performance by Gaussian Restricted Boltzmann Machines (GRBMS). Zhao-Rong Lai et al., [10] proposed the Discriminative and Compact Coding for Robust Face Recognition. Discriminative and Compact Coding (DCC) is introduced for multiple error measurements into regression models. There are two types of proposed models viz., (i) Multi-scale error measurements. (ii)Inspire within-class collaborative representation. DCC is robust method to produce the stable regression residual, which is more important for classification. Xiaobing Pei et al.,[11] proposed Manifold Adaptive Label Propagation (MALP) for Face Clustering. The model deals with the semi-supervised clustering problems. MALP tries to find graph weight matrix along with the graph edges of the given data set and integrates sparse representation constraint into regular framework of Label Propagation (LP), which can enhance the performance of LP in face clustering problem. The algorithm is tested on datasets like ORL, YALE, Extended YALEB and PIE. Randa Atta and Ghanbar [12] Proposed An Efficient Face Recognition System Based on Embedded DCT Pyramid. Each face image is decomposed into non-overlapping blocks of approximation sub bands and a set of reversed L-shape blocks with high frequency coefficients in the detailed sub bands. Inter dependency among the sub bands are computed using composite spatial orientation tree of the blocks and efficient coefficients of the DCT pyramid is selected by Set Partitioning in Hierarchal Trees (SPIHT). These coefficients are used as the features for the face recognition. Nearest Neighbor and Euclidean distance methods are used to classify the similar images of the database and test images.

\section{PROPOSED MODEL}

In this section the face recognition model is discussed based on novel concept of converting many images of single person into one image and features are extracted using DWT and SVM. The block diagram of the model is shown in Figure 1.

\subsection{Face Databases}

The various databases such as ORL, JAFFE, Indian Male, Indian Female and L-speck are used to test the model for performance analysis.

\subsubsection{Olivetti Research Laboratory (ORL) [13]}

The database has forty persons with ten images per person i.e., it has four hundred images in total. The face images are captured with different lighting conditions, facial expressions, timings and angles, the face image samples of a person are shown in Figure 2, with each image of $92 * 112$.

\subsubsection{Japanese Female Facial Expressions (JAFFE) [14]}

The database consists of ten persons with twenty images per person i.e., it has two hundred images in the database. The images are captured based on expressions such as emotional, happy, disgust, angry, natural movement, surprise etc. Each image is of size $256 * 256$ with grey scale format. The face image samples are shown in Figure 3. 
Signal \& Image Processing : An International Journal (SIPIJ) Vol.7, No.3, June 2016

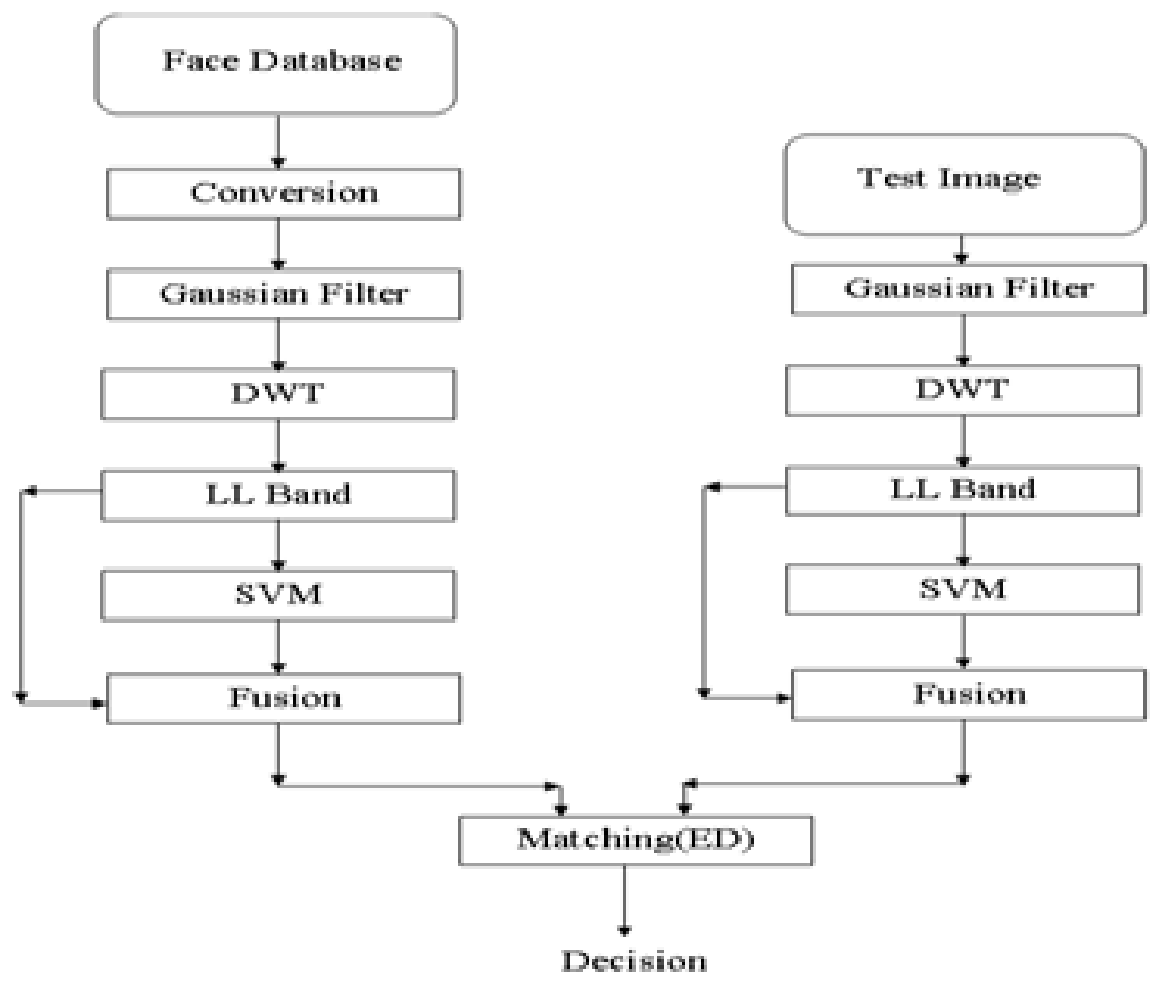

Figure 1. Proposed Model

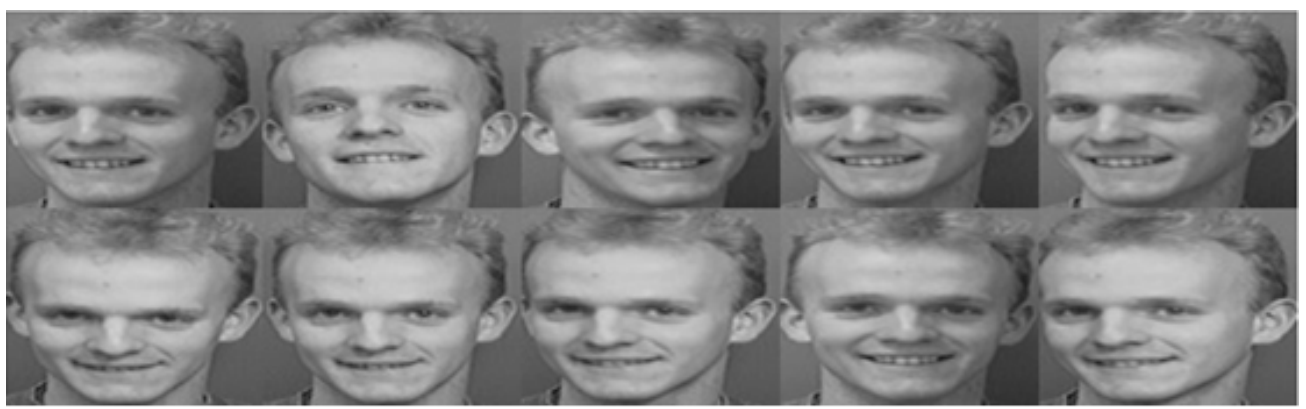

Figure 2. Samples of ORL face images

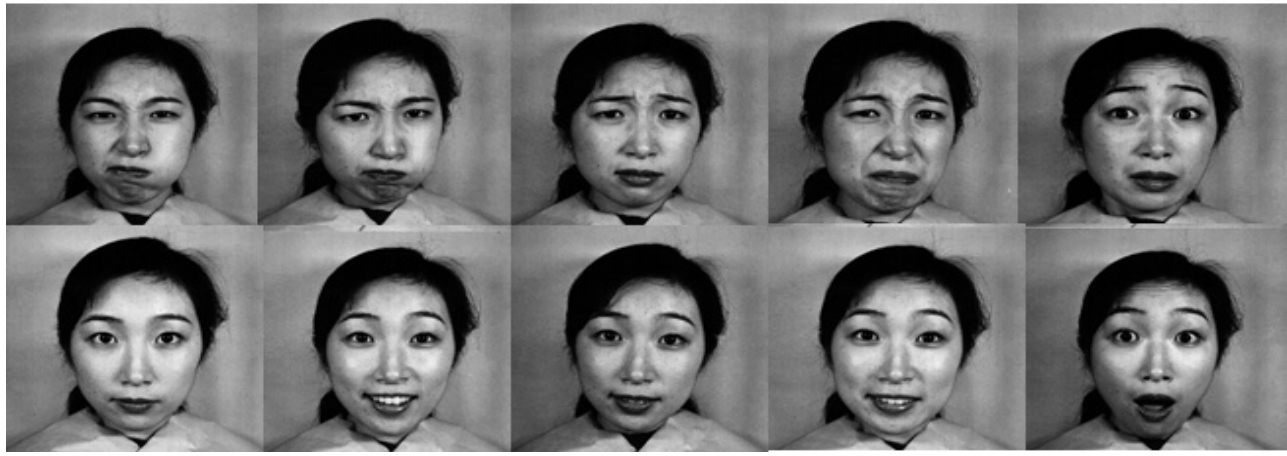

Figure 3. Samples of JAFFE images 


\subsubsection{Indian Female [15]}

The database has eleven persons with twenty two images per person i.e., it has two hundred forty images in total. The images of size $640 * 480$ are captured based on facial orientations with different emotional expressions. The samples of images are shown in Figure 4.

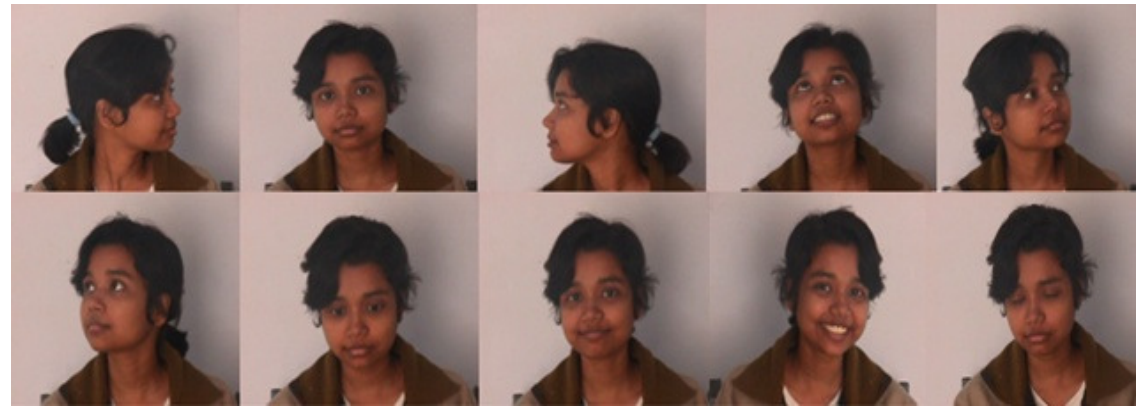

Figure 4. Samples of Indian Female images

\subsubsection{Indian Male [15]}

The face database has twenty persons with eleven images per persons. The face images are captured on different orientation angles with various expressions. Each image is of size $640 * 480$ and the face image samples are shown in Figure 5.

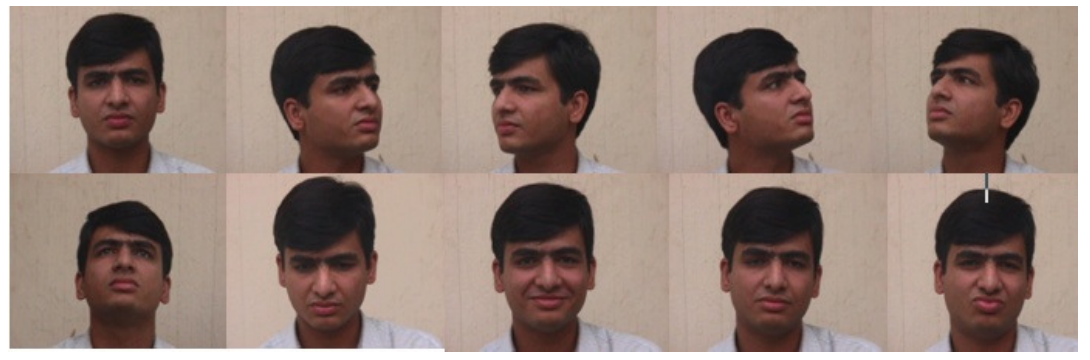

Figure 5. Samples of Indian Male images

\subsubsection{L-Speck Face Database [16]}

The database has one hundred and twenty persons with nineteen images per person ie., it has two thousand two hundred and eighty images. The database has image size of $320 * 280$ with BMP format. The images are captured with different expressions of a person. The samples of face images are shown in the Figure 6.

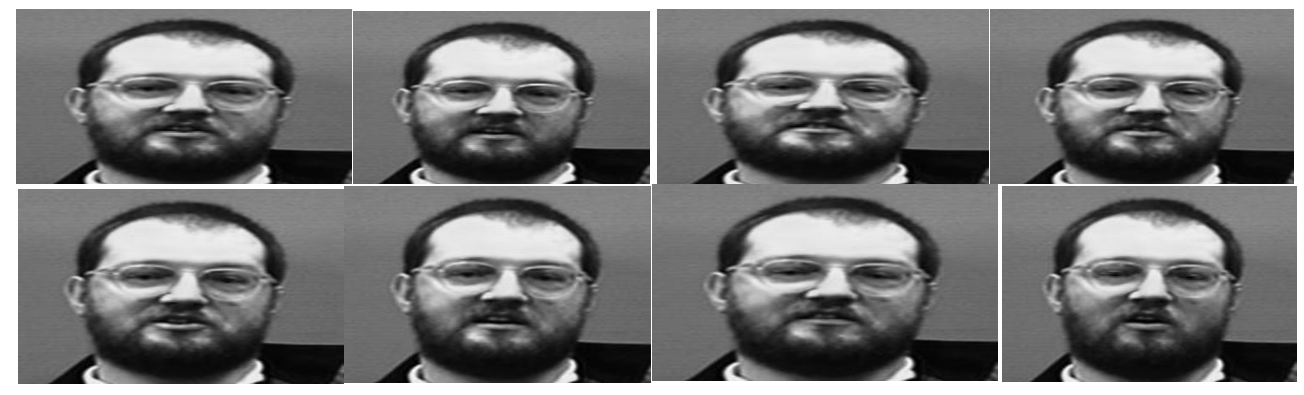

Figure 6. Samples of L-Speck Face Database images 


\subsection{Translation of images}

The number of image samples per person is converted into single image per person to save execution time and memory. The average intensity values of all images are used to derive single image per person. Eight images of single person are converted into single image as shown in Figure 7.

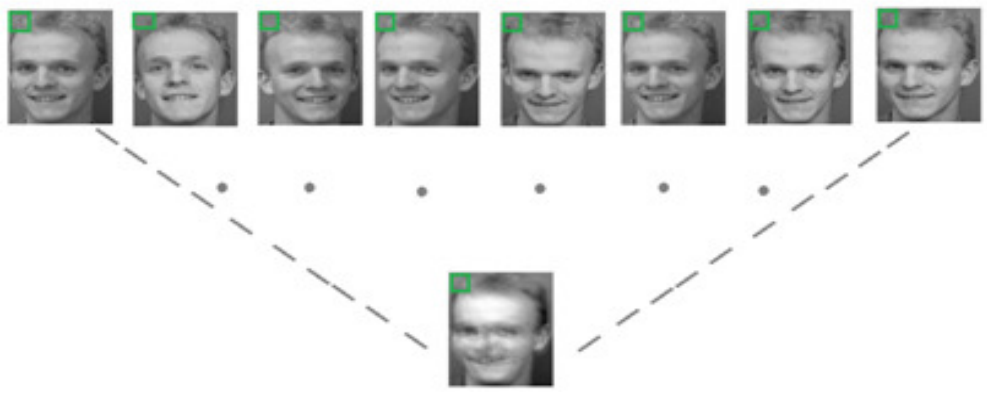

Figure 7. Translation of face images samples

\subsubsection{Demonstration of conversion using $4 * 4$ matrices}

The four $4 * 4$ matrices are considered as shown in Figure 8 to demonstrate the compression.

\begin{tabular}{|c|c|c|c|}
\hline$\left[\begin{array}{llll}10 & 12 & 16 & 98\end{array}\right]$ & {$\left[\begin{array}{llll}17 & 16 & 15 & 85\end{array}\right.$} & {$\left[\begin{array}{llll}12 & 19 & 0 & 100\end{array}\right]$} & 1229 \\
\hline 55693387 & $\begin{array}{llll}56 & 29 & 83 & 107\end{array}$ & 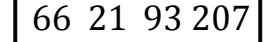 & $\begin{array}{llll}36 & 2 & 32 & 79\end{array}$ \\
\hline 3567 & $\begin{array}{llll}25 & 64 & 95 & 7\end{array}$ & $\begin{array}{llll}62 & 84 & 35 & 0\end{array}$ & $\begin{array}{llll}32 & 98 & 5 & 100\end{array}$ \\
\hline 219878 & {$\left[\begin{array}{llll}45 & 21 & 98 & 78\end{array}\right.$} & {$\left[\begin{array}{llll}95 & 41 & 38 & 18\end{array}\right]$} & 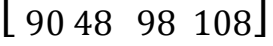 \\
\hline
\end{tabular}

Figure 8. Four $4 * 4$ Matrix for translation

Each $4 * 4$ matrices are converted into column vectors as shown in Figure 9. The four column vectors are converted into single column vector by computing element by element average values as shown in Figure 10(a). The column vector is converted into matrix as shown in Figure 10(b). The translation of many images of a person into single image is always an advantage of less execution time of an algorithm in real time and the memory required to one image is less compared to many images per person. The mathematical model to convert many images into one image is given in equation 1 .

$\left[\begin{array}{l}10 \\ 55 \\ 25 \\ 45 \\ 12 \\ 69 \\ 24 \\ 21 \\ 16 \\ 33 \\ 35 \\ 98 \\ 98 \\ 87 \\ 67 \\ 78\end{array}\right]\left[\begin{array}{c}17 \\ 56 \\ 25 \\ 45 \\ 16 \\ 29 \\ 64 \\ 21 \\ 15 \\ 83 \\ 95 \\ 98 \\ 85 \\ 107 \\ 07 \\ 78\end{array}\right] \quad\left[\begin{array}{c}12 \\ 66 \\ 62 \\ 95 \\ 19 \\ 21 \\ 84 \\ 41 \\ 00 \\ 93 \\ 35 \\ 38 \\ 100 \\ 207 \\ 00 \\ 18\end{array}\right] \quad\left[\begin{array}{c}01 \\ 36 \\ 32 \\ 90 \\ 22 \\ 02 \\ 98 \\ 48 \\ 09 \\ 32 \\ 05 \\ 98 \\ 11 \\ 79 \\ 100 \\ 108\end{array}\right]$

Figure 9. Column Vector of $4 * 4$ matrices 
Signal \& Image Processing : An International Journal (SIPIJ) Vol.7, No.3, June 2016

Consider an image size of $m^{*} n$ and convert into single column vector of size $\left(\left(\mathrm{m}^{*} \mathrm{n}\right), 1\right)$. The total numbers of column vectors are equivalent to total number of images per person. The average values of all column vectors are computed using equation 1 .

$$
X_{\text {avg }}=\left[\begin{array}{c}
X_{\text {avg } 1} \\
X_{\text {avg } 2} \\
X_{\text {avg } 3} \\
\cdot \\
\cdot \\
\cdot \\
\cdot \\
\cdot \\
\cdot \\
\cdot \\
\cdot \\
\cdot \\
X_{\text {avg }(m * n)}
\end{array}\right]=\left[\begin{array}{c}
10 \\
53.25 \\
36 \\
68.75 \\
17.25 \\
30.25 \\
67.5 \\
32.75 \\
10 \\
60.25 \\
42.50 \\
83 \\
73.50 \\
107.5 \\
43.5 \\
70.5
\end{array}\right] \quad X_{\text {avg }}=\left[\begin{array}{cccc}
10 & 17.25 & 10 & 73.5 \\
53.25 & 30.24 & 60.25 & 120 \\
36 & 67.5 & 42.5 & 43.5 \\
68.75 & 32.75 & 83 & 70.5
\end{array}\right]
$$

(a)

(b)

Figure 10. Single column vector and corresponding matrix

$$
X_{\text {avg }}=\left[X_{\operatorname{avg}(i)}\right]=\frac{\sum_{j=1}^{N} x(i, j)}{N}, \quad 1 \leq i \leq(m * n)
$$

For $i=1$,

$X_{\text {avg } 1}=\frac{\sum_{j=1}^{N} x(1, j)}{N}=\frac{x(1,1)+x(1,2)+\ldots \ldots \ldots+x(1,8)}{8}$

For $i=2$,

$X_{\text {avg } 2}=\frac{\sum_{j=1}^{N} x(2, j)}{N}=\frac{x(2,1)+x(2,2)+\ldots \ldots \ldots+x(2,8)}{8}$

For $i=m^{*} n$,

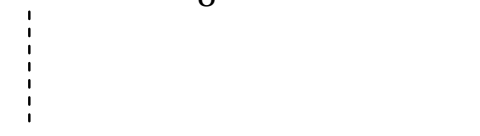

$X_{\operatorname{avg}(m * n)}=\frac{\sum_{j=1}^{N} x(m * n, j)}{N}=\frac{x(m * n, 1)+x(m * n, 2)+\ldots \ldots \ldots+x(m * n, 8)}{8}$

Where:

$$
\begin{aligned}
\mathrm{N} & =\text { Number of images per person } \\
\mathrm{m} & =\text { Number of rows in a matrix } \\
\mathrm{n} & =\text { Number of columns in a matrix }
\end{aligned}
$$

The column vector of $X_{\text {avg }}$ is converted back to $m * n$ image to compress many images to one image. 


\subsection{Gaussian Filter}

The Gaussian smoothing operator is a 2-D convolution operator i.e., used to blur images and remove detail noise. The Gaussian filter function is given by equation (3) with mean distribution of zero.

$G(x, y)=\frac{1}{2 \pi \sigma^{2}} e^{-\frac{x^{2+y^{2}}}{2 \sigma^{2}}}$

Where, $\sigma$ is the standard deviation of the distribution.

Gaussian filters are linear low pass filters which masks perfectly simulate optical blur and remove the details of the image. The degree of the smoothing is controlled by the $\sigma$, larger the $\sigma$ smoothing will be more. In the spatial domain the image is multiplied by appropriate kernel but in the frequency domain an image and a filter function are multiplied pixel by pixel to obtain the filtered output in Figure 11.

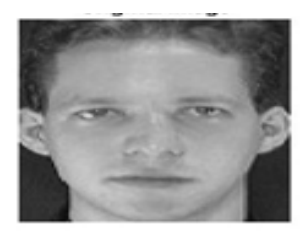

(a) Original image

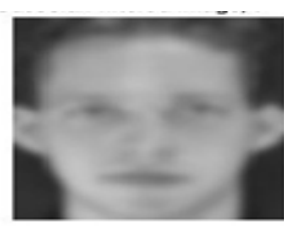

(b) Gaussian filtered image, $\sigma=2$

Figure11. Gaussian filter image

\subsection{Discrete Wavelet Transform [17]}

The transformation is used to obtain frequency resolution and temporal resolution for low and high frequencies respectively. The DWT divides an image into approximation and detailed sub bands as shown in figure 12. The approximation sub band has significant information of an image. The detailed sub band has information on horizontal, vertical and diagonal details. The low pass filters and high pass filters are used to generate approximation and detailed sub bands respectively. The four sub bands such as LL band formed by low pass filter and low pass filter, LH band formed by low pass and high pass filter, HL band is formed by high pass filter and low pass filter, $\mathrm{HH}$ band is formed by high pass filter and high pass filters.

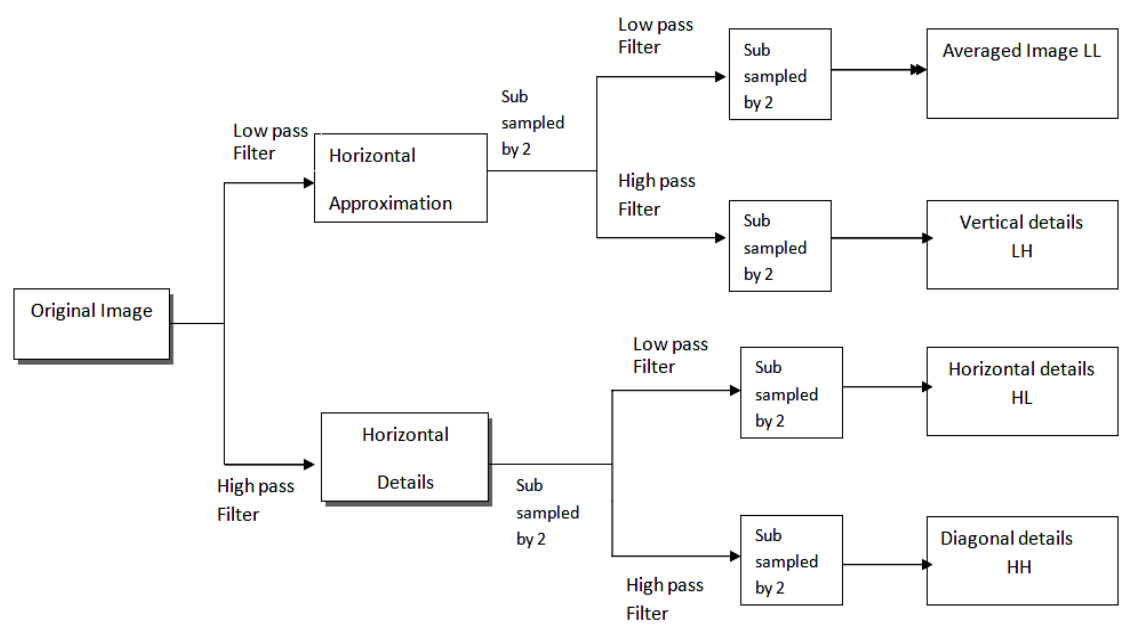

Figure 12. One level 2D-DWT 


\subsection{Support Vector Machine [18]}

It is used to find the position of the feature vectors, image compression and to classify the data. The support vectors are the data points located near to the hyper-plane shown in figure 13.

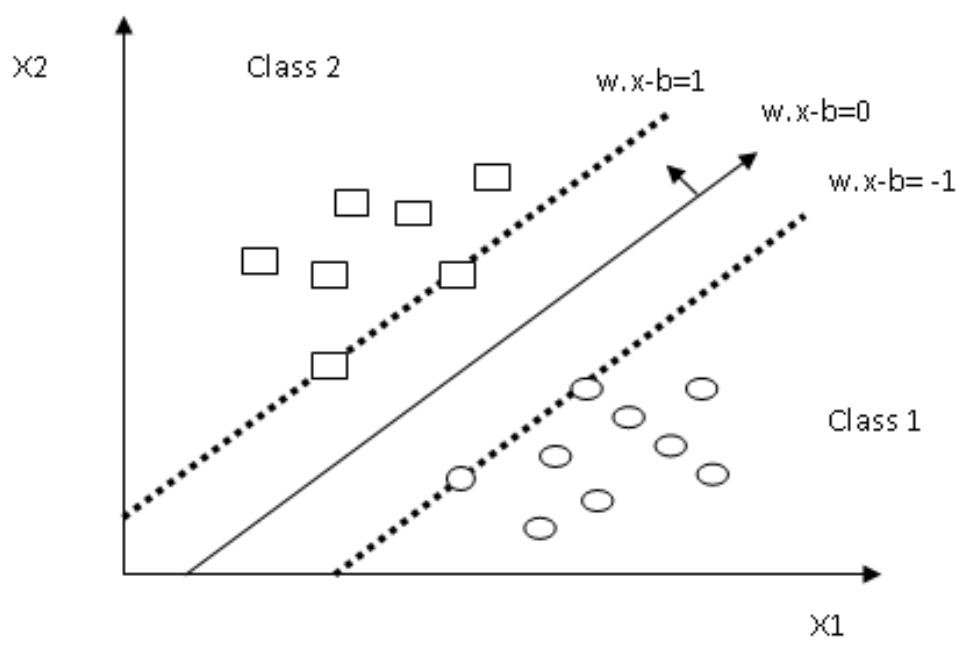

Figure13. SVM classification

The classifier divides into two groups using hyper-plane as class 1 and class 2 i.e., plus plane is $\mathrm{X}: \mathrm{W} \cdot \mathrm{X}+\mathrm{b}=1$ and minus plane is $\mathrm{X}: \mathrm{W} \cdot \mathrm{X}+\mathrm{b}=-1$. Where the $W$ is the weight vector and it is perpendicular to the hyper-plane, $X$ is the feature vector and $b$ is the position of the feature vector. The proposed method uses SVM for data compression. The support vectors of LL band coefficients are considered and fused with LL band coefficients to derive final features.

\subsection{Euclidean-Distance}

The final features of test images are compared with final features of images in the data base using Euclidian Distance (ED) to identify a person using equation (4).

$$
\mathrm{ED}=\sqrt{\sum_{i=1}^{M}(\mathrm{Pi}-\mathrm{qi}) 2}
$$

Where, $M=$ No of coefficients in a vector.

$\mathrm{Pi}=$ Coefficients values of vectors in database.

$\mathrm{q} i=$ Coefficient values of vectors in test image

\section{Algorithm}

Problem Definition: The novel face recognition algorithm is developed based on compression of spatial domain images and final features are generated using fusion of LL band co-efficients of DWT and Support Vectors of LL band to save memory and execution time. The algorithm is as shown in Table 1.

Objectives: The face recognition is used

(i) To identify a person effectively.

(ii) To reduce errors viz., FRR, FAR and EER.

(iii) To increase the value of TSR. 
Signal \& Image Processing : An International Journal (SIPIJ) Vol.7, No.3, June 2016

Table 1: Proposed Algorithm.

Input: Face database

Output: Identification of a person

1. The face database is created

2. Many face images of single person are converted into single image using averaging technique

3. Gaussian filter is used to extract better features.

4. The DWT is applied on filtered output face image.

5. The SVM is trained using LL band coefficients of DWT.

6. The LL band coefficients of DWT and SV's are fused based on arithmetic addition to generate final feature set.

7. The ED is used to compare final features of face database and test face images to compute performance parameters.

\section{Performance Analysis}

In this section, the definitions of performance parameters and result analysis based on TSR, FRR, FAR and EER are discussed.

\subsection{Definitions of Performance Parameters}

\subsubsection{False Rejection Ration (FRR):}

The ratio of number of genuine persons rejected to the total number of persons inside the database as given in the equation 5 .

$\mathrm{FRR}=\frac{\text { Number of genuine persons in the database rejected }}{\text { Total number of persons inside the database }}$

5.1.2 False Acceptance Ratio (FAR): The ratio of number of imposer persons accepted as genuine to the total number of persons outside the database as given in equation 6 .

$\mathrm{FAR}=\frac{\text { Number of imposter } \text { persons accepted as genuine }}{\text { Total number of persons outside the database }}$

5.1.3 Total Success Rate (TSR): The ratio of number of genuine persons recognized correctly to the total number of persons inside the database as given in equation 7 .

$\mathrm{TSR}=\frac{\text { Number of the genuine persons recognized correctly }}{\text { Total number of the persons inside the database }}$

5.1.4 Equal Error Rate (EER): The optimum error between the FAR and FRR. The value of EER is intersection of FRR and FAR. The lower EER gives the better efficiency of an algorithm.

\subsection{Performance Evaluation using Various Face Databases}

5.2.1 ORL Face Database: The database is created by considering ten Persons Inside the Database (PID) and thirty Persons Outside the Database (POD) to observe the variation of FRR, FAR, TSR and EER for threshold value variations of the proposed model. The variations of FRR, FAR and TSR with the threshold values are shown in the Figure 14. 
Signal \& Image Processing : An International Journal (SIPIJ) Vol.7, No.3, June 2016

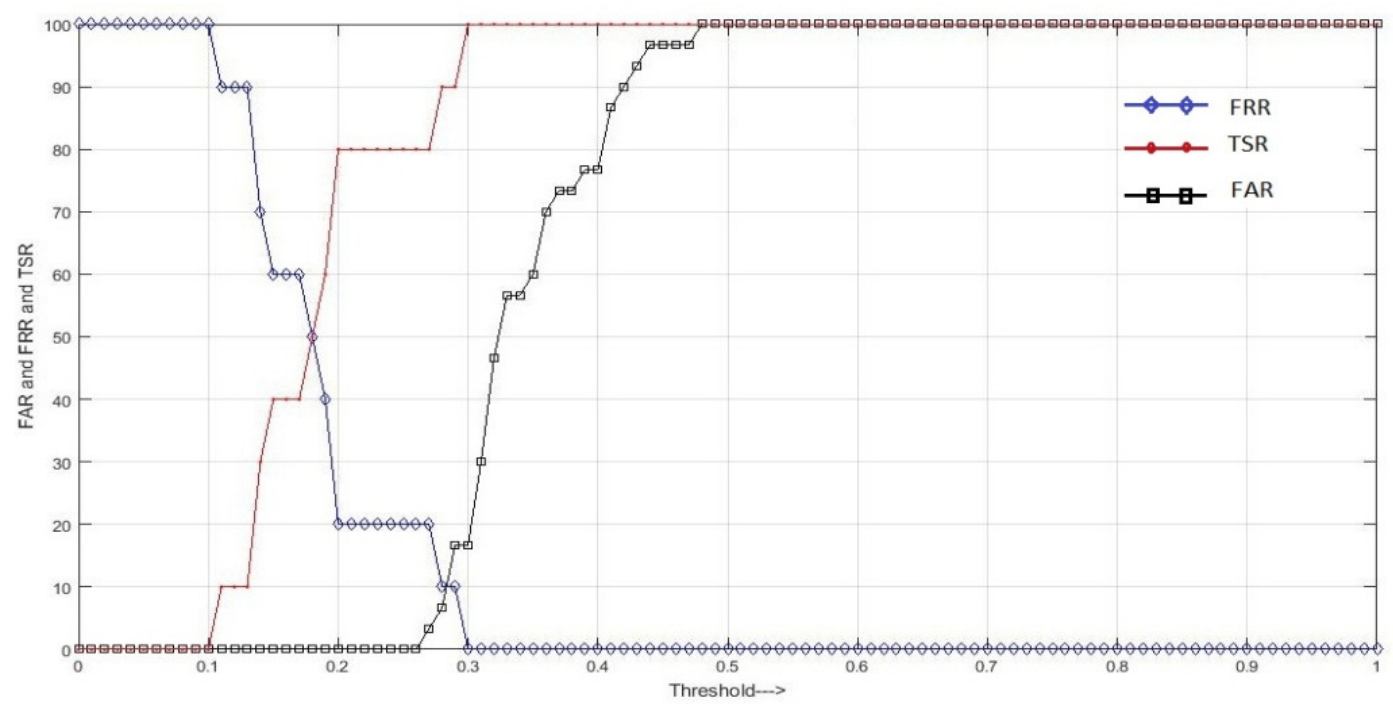

Figure14. The variations of performance parameter with Threshold

The performance values of FAR and TSR increases with threshold, where as the percentage FRR values decreases with threshold. It is observed that the percentage TSR value is $90 \%$ corresponding to $10 \%$ EER and maximum TSR is $100 \%$.The variations of percentage EER ,optimum TSR and maximum TSR for different combinations of the PID \& POD for ORL face database is given in Table 2. It is observed that, the values of the optimum TSR decreases and EER increases as PID increases. The optimum TSR values are directly proportional to POD, where as the values of EER are inversely proportional to POD.

Table 2.Variations of performance parameters with PID and POD for ORL database

\begin{tabular}{|c|c|c|c|c|}
\hline & POD & Opt TSR (\%) & Max TSR (\%) & EER (\%) \\
\hline 10 & 30 & 90 & 100 & 10 \\
\hline 16 & 24 & 81.25 & 93.7 & 18.75 \\
\hline 20 & 20 & 80 & 100 & 20 \\
\hline
\end{tabular}

5.2.2 JAFFE Face Database: The database is created by considering ten persons inside the database and thirty persons outside the database to observe the variations of FRR, FAR, TSR and EER for different threshold values. The variations of FRR, FAR and TSR with the threshold values are shown in the Figure 15. 
Signal \& Image Processing : An International Journal (SIPIJ) Vol.7, No.3, June 2016

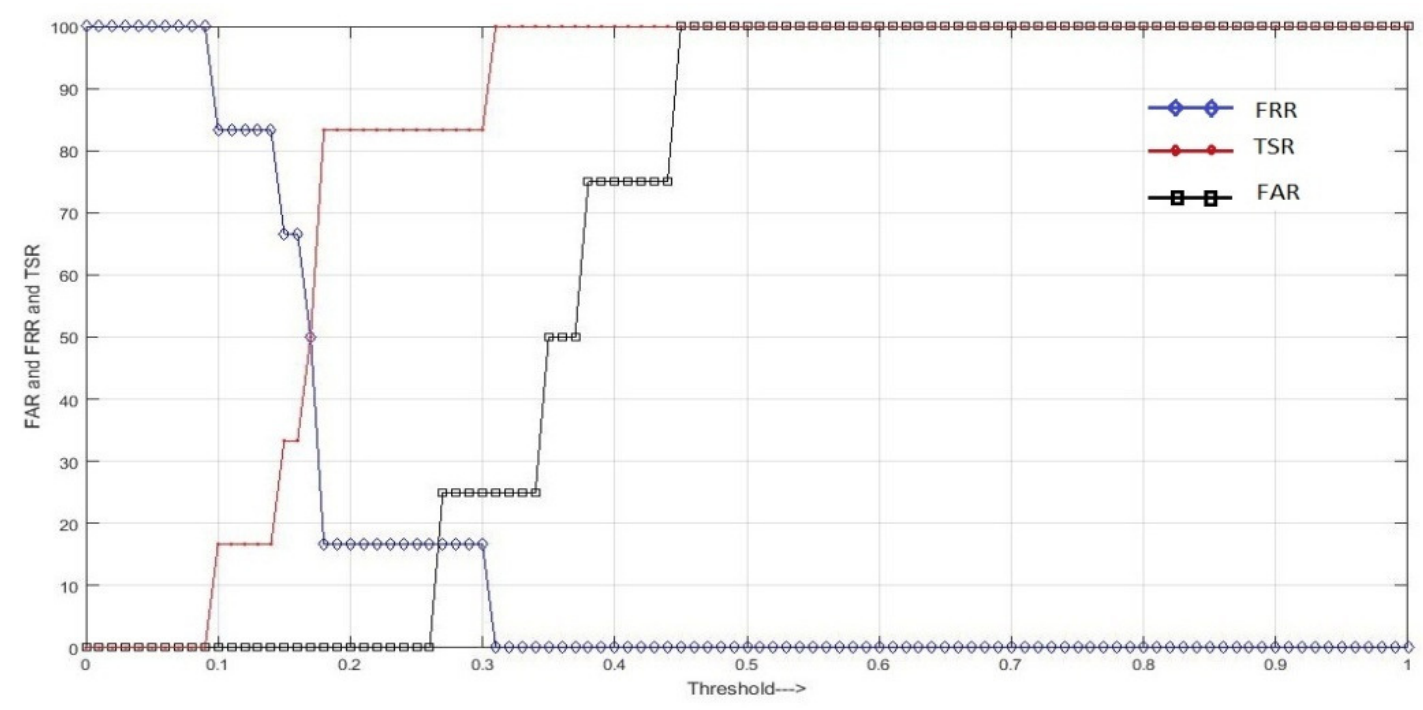

Figure15. The variations of performance parameter with threshold

The performance values of FAR and TSR increases with threshold, where as the percentage FRR values decreases with threshold. It is observed that the percentage TSR value is $83.33 \%$ corresponding to $17 \%$ EER and maximum TSR is $100 \%$. The variations of percentage EER ,optimum TSR and maximum TSR for different combinations of the PID \& POD for JAFFE face database is given in Table 3. It is observed that, the values of the optimum TSR decreases and EER increases as PID decreases. The optimum TSR values are inversely proportional to POD, whereas the values of EER are directly proportional to POD.

Table 3.Variations of performance parameters with PID and POD for JEFFE database

\begin{tabular}{|c|c|c|c|c|}
\hline PID & POD & Opt TSR (\%) & Max TSR (\%) & EER (\%) \\
\hline 6 & 4 & 83.33 & 100 & 17 \\
\hline 5 & 5 & 80 & 100 & 20 \\
\hline
\end{tabular}

\subsubsection{Indian Male Database:}

The database is created by considering ten persons inside the database and thirty persons outside the database to observe the variations of FRR, FAR, TSR and EER for threshold values. The variation of FRR, FAR and TSR with the threshold values are shown in the Figure 16. 
Signal \& Image Processing : An International Journal (SIPIJ) Vol.7, No.3, June 2016

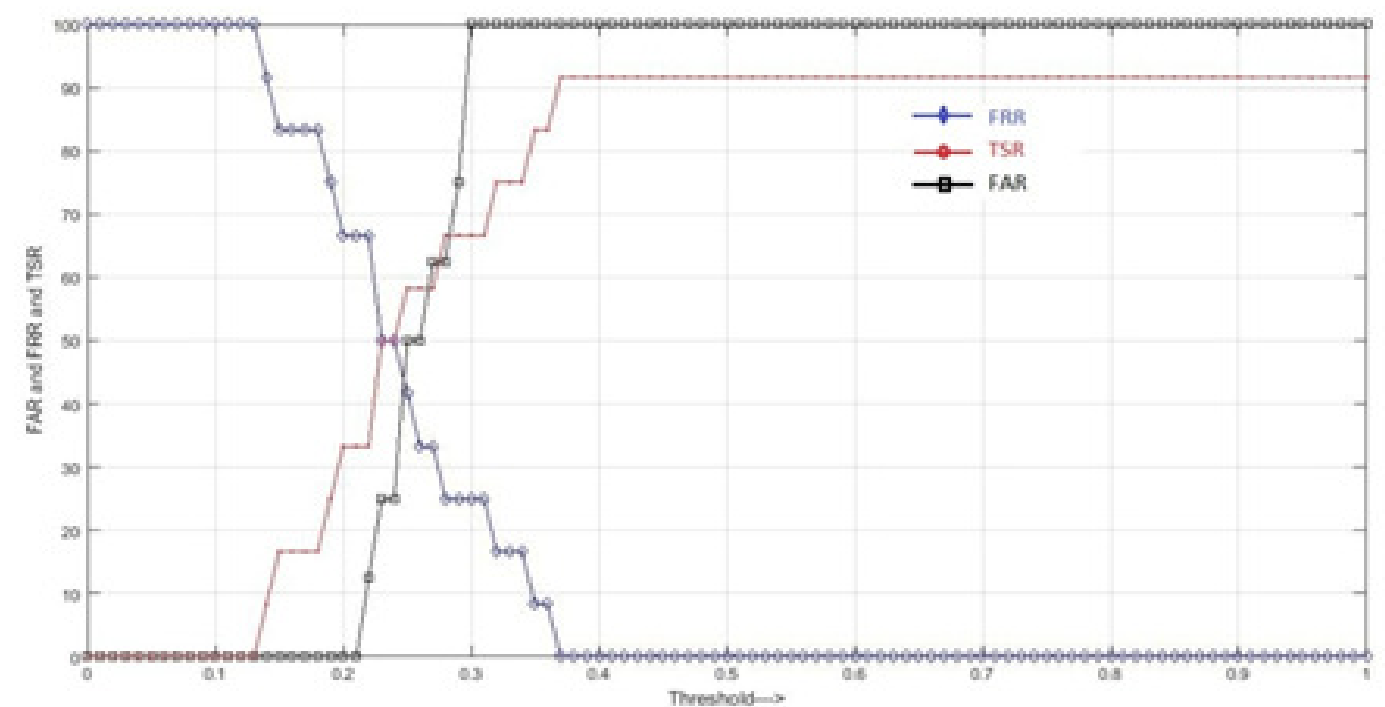

Figure 16: The variations of performance parameters with threshold

The performance values of FAR and TSR increases with threshold, whereas the percentage FRR values decreases with threshold. It is observe that the percentage TSR value is $58.33 \%$ corresponding to $44 \%$ EER and maximum TSR is $91.667 \%$. The variations of percentage EER, optimum TSR and maximum TSR for different combinations of the PID \& POD for Indian Male face database is given in Table 4.It is observed that, the values of the optimum TSR increases and EER decreases as PID increases. The optimum TSR values are inversely proportion to POD, where as the values of EER are directly proportional to POD.

Table 4.Variations of performance parameters with PID and POD for Indian Male database

\begin{tabular}{|c|c|c|c|c|}
\hline PID & POD & Opt TSR (\%) & Max TSR (\%) & EER (\%) \\
\hline 5 & 5 & 20 & 100 & 80 \\
\hline 6 & 14 & 33.33 & 100 & 60 \\
\hline 8 & 12 & 50 & 100 & 50 \\
\hline 12 & 8 & 58.33 & 91.667 & 44 \\
\hline
\end{tabular}

\subsubsection{Indian Female Database:}

The database is created by considering ten persons inside the database and thirty persons outside the database to observe the variations of FRR, FAR, TSR and EER for different threshold values. The variations of FRR, FAR and TSR with the threshold values are shown in the Figure 17. 
Signal \& Image Processing : An International Journal (SIPIJ) Vol.7, No.3, June 2016

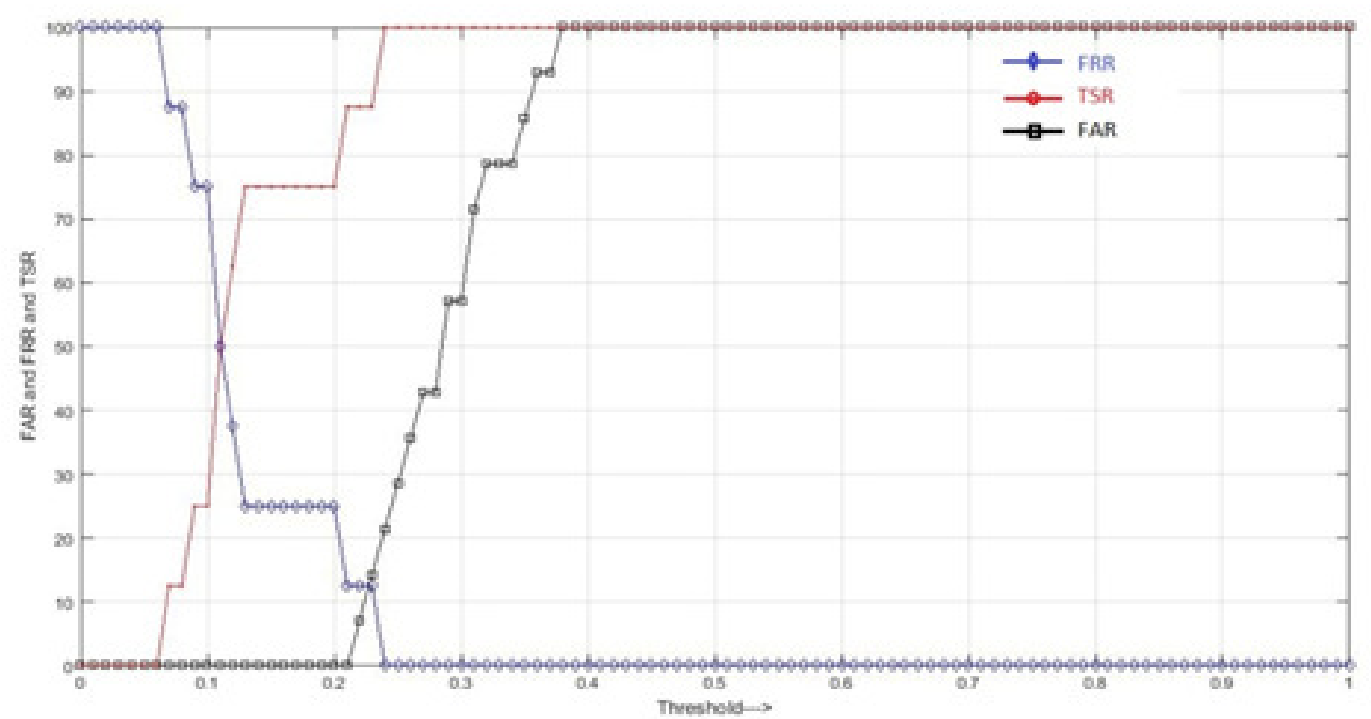

Figure17. The variations of performance parameters with Threshold

The performance values of FAR and TSR increases with threshold, whereas the percentage FRR values decreases with threshold. It is observed that the percentage TSR value is $87.5 \%$ corresponding to $12.5 \%$ EER and maximum TSR is $100 \%$. The variations of percentage EER, optimum TSR and maximum TSR for different combinations of the PID \& POD for Indian Female face database is given in Table 5. It is observed that, the values of the EER increases as PID increases. The optimum TSR values are inversely proportional to POD, where as the values of EER are inversely proportional to POD.

Table5. Variations of performance parameters with PID and POD for Indian female database

\begin{tabular}{|c|c|c|c|c|}
\hline PID & POD & Opt TSR (\%) & Max TSR (\%) & EER (\%) \\
\hline 6 & 16 & 83.33 & 100 & 10 \\
\hline 8 & 14 & 87.5 & 100 & 12.5 \\
\hline 12 & 10 & 83.33 & 100 & 16.66 \\
\hline
\end{tabular}

\subsection{L-Speck Face Database:}

The database is created by considering ten persons inside the database and thirty persons outside the database to observe the variations of FRR, FAR, TSR and EER for different threshold values. The variations of FRR, FAR and TSR with the threshold values are shown in the Figure 18. 
Signal \& Image Processing : An International Journal (SIPIJ) Vol.7, No.3, June 2016

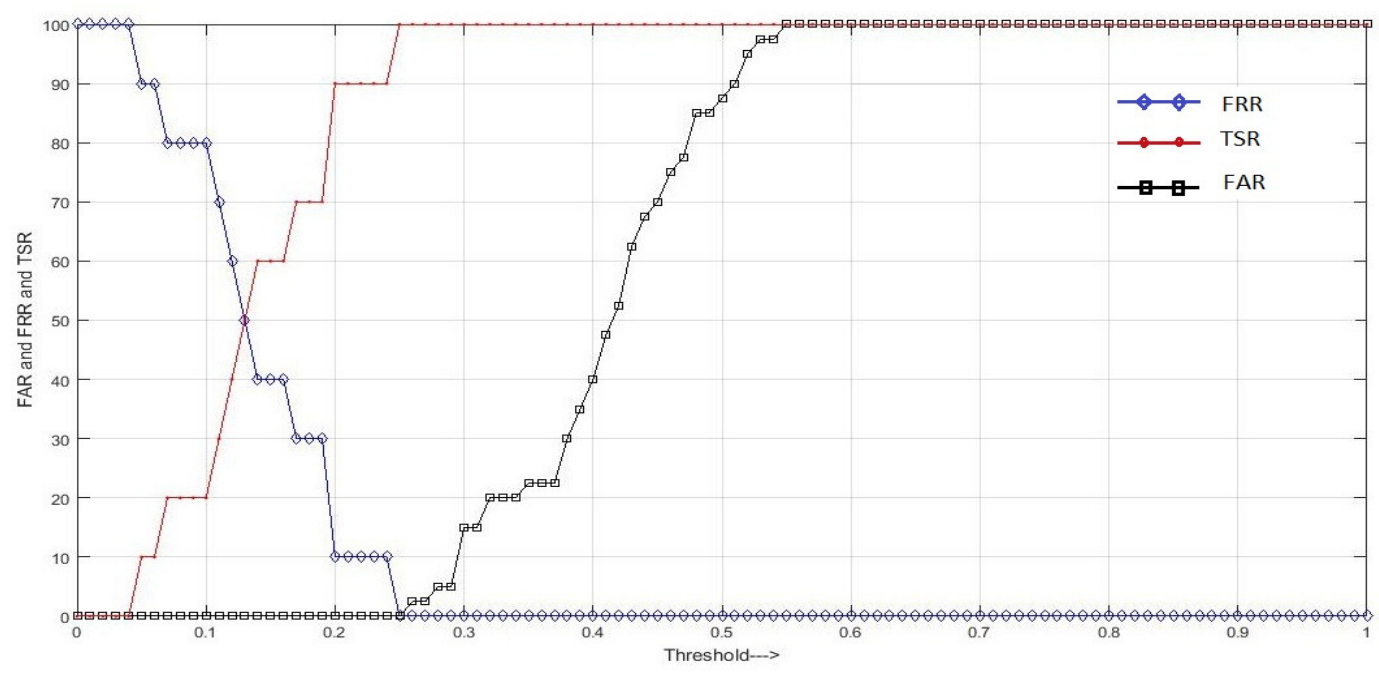

Figure 18. The variations of performance parameters with threshold

The performance values of FAR and TSR increases with threshold, whereas the percentage FRR values decreases with threshold. It is observed that the percentage TSR value is $100 \%$ corresponding to $0 \%$ EER and maximum TSR is $100 \%$. The variations of percentage EER, optimum TSR and maximum TSR for different combinations of the PID \& POD for L-Speck face database is given in Table 6. It is observed that, the values of the optimum TSR decreases and EER increases as PID increases. The optimum TSR values are directly proportion to POD, whereas the values of EER are inversely proportional to POD.

Table 6.Variations of performance parameters with PID and POD for L-Speck database

\begin{tabular}{|c|c|c|c|c|}
\hline PID & POD & Opt TSR (\%) & Max TSR (\%) & EER (\%) \\
\hline 10 & 40 & 100 & 100 & 0 \\
\hline 20 & 30 & 90 & 95 & 0.066 \\
\hline 30 & 20 & 90 & 93.33 & 0.08 \\
\hline
\end{tabular}

\subsection{Comparison of Proposed algorithm with Existing algorithms for ORL Face database}

The performance parameters viz., optimum TSR and maximum TSR of the proposed method is compared with existing methods presented by D Murugan et al.,[19] Pallavi D.Wadkar et al.,[20] and Sujatha B M et al.,[21] is shown in Table 7. It is observed that the values of the optimum TSR and maximum TSR values are high in the case of proposed algorithm compared to existing algorithms. The proposed algorithm is superior compared to existing algorithms for the following reasons. 
Signal \& Image Processing : An International Journal (SIPIJ) Vol.7, No.3, June 2016

(i) The novel concept of converting many images per person into single image using averaging technique.

(ii)The SVM is applied on LL band of DWT and the support vectors (SV's) are considered.

(iii)The LL band coefficients are fused with SV's to generate final feature set.

(iv)The algorithm is very useful as the images are compressed based on Image averaging technique, DWT and SVM.

Table 7. Comparison of performance parameters of proposed method with existing methods

\begin{tabular}{|c|c|c|c|c|}
\hline SL. No. & Authors & Technique used & Opt TSR(\%) & Max TSR (\%) \\
\hline 1 & D Murugan et al [19]., & Gabor filter+DWT & 84.8 & 92 \\
\hline 2 & Pallavi D.Wadkar et al [20]., & DWT & 82.85 & 90 \\
\hline 3 & Sujatha B M et al [21]., & DWT+FFT+CLBP & 80 & 93.33 \\
\hline 4 & Proposed Model & DWT+SVM & $\mathbf{9 0}$ & $\mathbf{1 0 0}$ \\
\hline
\end{tabular}

\section{CONCLUSION}

Face recognition is an effective biometric to identify a person. In this paper, we proposed compression based face recognition using DWT and SVM. Many images of a person are compressed to one image per person using averaging technique. The DWT is applied on face images to compress it by considering only approximation i.e., LL band only. The LL band coefficients are applied to SVM to generate SV's. The LL band coefficients and SV's are fused to derive final features. The ED is used to compare database features with test image features to test the performance of an algorithm. It is observed that, the performance of the proposed method is better compared to existing methods. In future, the algorithm can be implemented using hardware.

\section{REFERENCES}

[1] Thai Hoang Le and Len Bui "Face Recognition based on SVM and 2DPCA" International Journal of signal processing, Image processing and Pattern Recognition, Vol. 4, No. 3, September, 2011

[2] Saeid Fazli, Siroos Toofan, and Zahra Mehrara,"JPEG2000 Image Compression using SVM and DWT”, International Journal of Science and Engineering Investigations, vol. 1, pp. 54-57, issue 3, April 2012.

[3] Harin Sellahewa and Sabah A. Jassim, "Image-Quality-Based Adaptive Face Recognition", IEEE Transactions on Instrumentation and Measurements, Vol. 59, No. 4, pp. 805-813. April 2010.

[4] Vinoda Yaragatti and Bhaskar B, "Face Recognition using Neural Networks", International Journal of Electrical, Electronics Engineering and Telecommunications, Vol. 3, pp. 123-130, No. 3, July 2014. 
Signal \& Image Processing : An International Journal (SIPIJ) Vol.7, No.3, June 2016

[5] Idan Ram, Israel Cohen, and Michael Elad, "Facial Image Compression using Patch-Ordering Based Adaptive Wavelet Transform”, IEEE Transaction on Signal Processing, Vol. 21, No. 10, pp. 12701274, October 2014.

[6] S. Grgic and M. Grgic, "Performance of Image Compression using Wavelet", IEEE Transaction on Industrial Electronics, Vol. 48, No. 3, pp. 682-695, June 2001.

[7] Michael Elad, Roman Goldenberg, and Ron Kimmel, "Low Bit-Rate Compression of Facial Images", IEEE Transaction on Image Processing, Vol. 16, No. 9, pp. 2379-2383, September 2007.

[8] Kai Dong-Hyuk Shin, Rae-Hong Park, Seungjoon Yang and Jae-Han Jung, "Block-Based Noise Estimation using Adaptive Gaussian Filtering", IEEE Transactions on Consumer Electronics, Vol. 51, No. 1, pp. 218-226, February 2005.

[9] Munawar Hayat, Mohammad Bennamoun and Senjian An, ” Deep Reconstruction Models for Image Set Classification “, IEEE Transactions on Pattern analysis and Machine Intelligence, pp.1-15, April 2014.

[10] Zhao-Rong Lai, Dao-Qing Dai, Chuan-Xian Ren and Ke-Kun Huang " Discriminative and Compact Coding for Robust Face Recognition" IEEE Transaction on Cybernetics, Vol. 45, No.9, pp.19001912, September 2015.

[11] Xiaobing Pei, Zehua Lyu, Changqing Chen and Chuanbo Chen "Manifold Adaptive Label Propagation for Face Clustering" IEEE Transactions on Cybernetics, Vol. 45, No.8, pp. 1681-1691, August 2015

[12] R. Atta and M. Ghanbar, "An Efficient Face Recognition System Based on Embedded DCT Pyramid", IEEE Transactions on Consumer Electronics, Vol. 58, No.4, pp.1285-1293, November 2012.

[13] ORL database, http://www.camrol.co.uk

[14] Jaffe Database, http://www.kasrl.org/jaffe_download.html.

[15] Indian Face Database, http://viswww.cs.umass.edu/ vidit/Indian Face Database.

[16] L-spacek databasehttp://cswww.essex.ac.uk/mv/allfaces

[17] Satish Bhairannawar, SayatamSarkar, Anand Y R, Raja K B and Venugopal K R, “An EfficientVLSI Architecture for Fingerprint Recognition using O2D-DWT architecture and Modified CORDICFFT”, IEEE International Conference on Signal Processing, Informatics Communication and Energy Systems, pp. 1-5, Calicut (NITC), 2015.

[18] Christopher J.C. Burges, “A Tutorial on Support Vector Machines for Pattern Recognition”, Kluwer Academic Publishers, pp. 1-43, 1998

[19] D Murugan, S Arumugam, K Rajalakshmi and Manish T, "Performance Evaluation of Face Recognition using Gabor Filter, Log Gabor filter and Discrete Wavelet Transform," International Journal of computer science and Information Technology, vol. 2, No.1, pp. 125-133, 2010.

[20] Pallavi D.Wadkar and Megha Wankhade, "Face Recognition using Discrete Wavelet Transforms," International Journal of Advanced Engineering Technology, vol. 3, pp. 239-242, 2012.

[21] Sujatha B M, K Suresh Babu, K B Raja, Venugopal K R, "Hybrid Domain based Face Recognition using DWT, FFT and Compressed CLBP" International Journal of Image processing (IJIP), Volume (9) , Issue (5) , pp. 283-303, 2015. 
Signal \& Image Processing : An International Journal (SIPIJ) Vol.7, No.3, June 2016

\section{AUTHORS}

Mrs. Sujatha BM is an Associate Professor in the Department of Electronics and Communication Engineering, Acharya Institute of Technology, Bangalore. She obtained her B.E degree in Electronics and Communication Engineering from Bangalore University and M.E degree in Electronics and Communication from University Visvesvaraya College of Engineering, Bangalore University, Bangalore. She is currently pursuing Ph.D in Computer Science and Engineering of Bangalore University under the guidance of Dr. K Suresh Babu, Professor, Department of Electronics and Communication Engineering, University Visvesvaraya College of

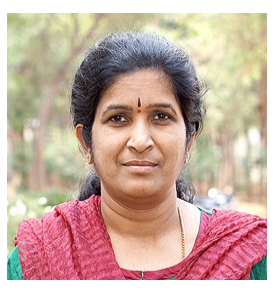
Engineering. She has over 15 research publications in refereed International Journals and Conference Proceedings. Her research interests include Image Processing, Biometrics, Computer Networks and Communication Engineering. She is a life member of Indian Society for Technical Education, New Delhi.

Mr. Chetan Tippanna Madiwalar obtained his B.Tech degree in Electronics and Communication Engineering from Manipal University and currently pursuing M.E degree in Electronics and Communication from University Visvesvaraya College of Engineering, Bangalore University, Bangalore. His research interests include Image Processing, Biometrics and VLSI.

K Suresh Babu is an Associate Professor, Dept. of Electronics and Communication Engineering, University Visvesvaraya College of Engineering, Ban galore University, Bangalore. He obtained his $\mathrm{BE}$ and $\mathrm{ME}$ in Electronics and Communication Engineering from University Visvesvaraya College of Engineering, Bangalore. He was awarded Ph.D. in Computer Science and Engineering from Bangalore University. He has over 30 research publications in refereed International Journals and Conference Proceedings. His research interests include Image Processing, Biometrics, and Signal Processing.

K B Raja is an Associate Professor, Dept. of Electronics and Communication Engineering, University Visvesvaraya College of Engineering, Bangalore University, Bangalore. He obtained his $\mathrm{BE}$ and $\mathrm{ME}$ in Electronics and Communication Engineering from University Visvesvaraya College of Engineering, Bangalore. He was awarded Ph.D. in Computer Science and Engineering from Bangalore University. He has over 160 research publications in refereed International Journals and Conference Proceedings. His research interests include Image Processing, Biometrics, VLSI Signal Processing and Computer Networks.
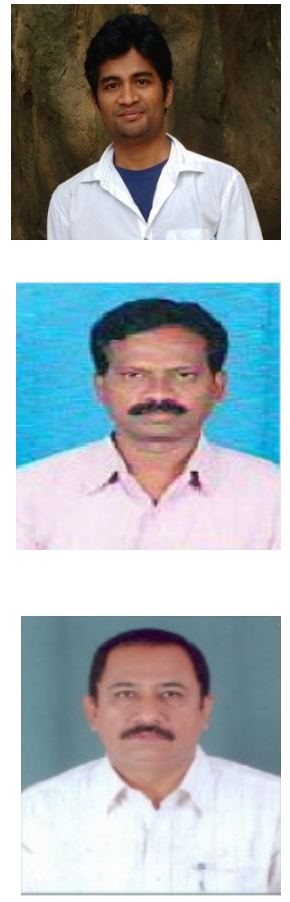

K R Venugopal is currently the Principal, University Visvesvaraya College of Engineering, Bangalore University, Bangalore. He obtained his Bachelor of Engineering from University Visvesvaraya College of Engineering. He received his Master's degree in Computer Science and Automation from Indian Institute of Science, Bangalore. He was awarded Ph.D. in Economics from Bangalore University and Ph.D. in Computer Science from Indian Institute of Technology, Madras. He has a distinguished academic career and has degrees in Electronics, Economics, Law, Business Finance, Public Relations, Communications, Industrial Relations, Computer Science and Journalism. He has authored 27 books on

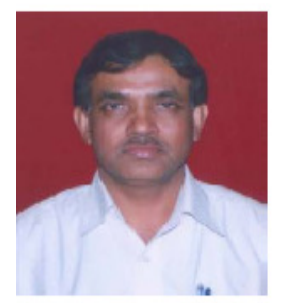
Computer Science and Economics, which include Petrodollar and the World Economy, C Aptitude, Mastering C, Microprocessor Programming, Mastering C++ etc. He has been serving as the Professor and Chairman, Department of Computer Science and Engineering, University Visvesvaraya College of Engineering, Bangalore University, Bangalore. During his three decades of service at UVCE he has over 375 research papers to his credit. His research interests include computer networks, parallel and distributed systems, digital signal processing and data mining. 